PROCEEDINGS OF THE

AMERICAN MATHEMATICAL SOCIETY

Volume 127, Number 10, Pages 2811-2820

S 0002-9939(99)05173-4

Article electronically published on April 23, 1999

\title{
AN APPLICATION OF THE REGULARIZED SIEGEL-WEIL FORMULA ON UNITARY GROUPS TO A THETA LIFTING PROBLEM
}

\author{
VICTOR TAN \\ (Communicated by Dennis A. Hejhal)
}

\begin{abstract}
Let $U(2)$ and $U(2,1)$ be the pair of unitary groups over a global field $F$ and $\pi$ an irreducible cuspidal representation of $U(2)$ which satisfies a certain $L$-function condition. By using a regularized Siegel-Weil formula, we can show that the global theta lifting of $\pi$ in $U(2,1)$ is non-trivial if every local factor $\pi_{v}$ of $\pi$ has a local theta lifting (Howe lifting) in $U(2,1)\left(F_{v}\right)$.
\end{abstract}

\section{INTRODUCTION}

In this paper, we will apply a regularized Siegel-Weil formula [Tan2] to a theta lifting problem for the dual pair of unitary groups $U(2)$ and $U(2,1)$ defined over a global field $F$. The motivation of this work come from [KR] and [KRS] which proved the regularized Siegel-Weil formula for the symplectic-orthogonal dual pair $S p(n), O(m)$ and applied it to poles and values of $L$-functions of $S p(n)$.

Given an irreducible cuspidal representation $\pi$ of $U(2)$, we would like to ask if its global theta lifting $\Theta(\pi)$ in $U(2,1)$, to be defined below, is non-trivial. Let $\bigotimes \pi_{v}$ be the decomposition of $\pi$ as a restricted tensor product where $v$ runs through all the places of $F$. We say that $\pi_{v}$ has a local theta lift (or Howe lift) to $U(2,1)\left(F_{v}\right)$ if $\pi_{v}$ occurs in the (local) Howe correspondence for the dual reductive pair. (See for example $[\mathrm{MVW}]$.

We will show that, under a certain condition on $\pi$, if $\pi_{v}$ has a local theta lifting everywhere, then $\pi$ has a global theta lifting $\Theta(\pi)$. More precisely,

Theorem 1.1. Let $\pi=\bigotimes \pi_{v}$ be a cuspidal representation of $U(2)$ such that $\pi_{v}$ belongs to the discrete series for all ramified places $v$ and the standard Langlands $L$-function $L\left(s, \pi, \gamma^{3}\right)$ has no pole at $s=1$. Then the global theta lifting $\Theta(\pi)$ of $\pi$ to $U(2,1)$ is non-trivial if and only if the local theta lifting of $\pi_{v}$ to $U(2,1)_{v}$ is nontrivial for all $v$.

All the notations and terminologies will be introduced in section 2 and the proof of the theorem will be given in section 4 .

We remark here that, for a general dual pair $U(m)$ and $U(n)$ such that $m>2 n$ (the convergent range), it has been proved in [Li2] that this is the case using the classical Siegel-Weil formula on $U(m)$ and $U(n, n)$. Note that this pair lies in the convergent range for the classical formula to work. Since our dual pair falls outside

Received by the editors December 2, 1997.

1991 Mathematics Subject Classification. Primary 11F70; Secondary 11F27, 22 E50.

(C)1999 American Mathematical Society 
this range, we have to resort to the regularized version of the Siegel-Weil formula which we will recall in section 3 .

This paper is based on part of the author's dissertation [Tan1] under the guidance of Jonathan Rogawski. This result was first announced in [GRS1] based on the approach in [Tan1]. The result was later improved in [GRS2] using a sophisticated arguments involving endoscopic $L$-packets. It should be mentioned that, in that paper, the hypothesis that $\pi_{v}$ belongs to the discrete series at all ramified places has been removed. However, this condition is needed in our approach in order to conclude the non-vanishing of certain local integrals defined in section 4 . We believe that the local theta lifting of $\pi_{v}$ should be enough to imply these local integrals are nonzero provided they converge. Nevertheless, our approach is more elementary and our emphasis is on the application of the Siegel-Weil formula.

The author expresses his sincere thanks to Professor Rogawski for suggesting this problem and providing many insightful comments. The author has also profited from direct conversation with Stephen Gelbart and Jian-Shu Li.

\section{SET UP}

Fix a quadratic extension $E$ of $F$. Let $G=U(2)$ act on the two-dimensional Hermitian space $U$ over $E$ with Hermitian form $($,$) and H=U(2,1)$ act on the three dimensional skew-Hermitian space $V$ over $E$ with skew-Hermitian form $(,)^{\prime}$. We form the tensor product $W=U \otimes_{E} V$ together with the symplectic form $\langle\rangle=,(,) \otimes \overline{(,)^{\prime}}$. Let $X \oplus Y$ be a complete polarization of $W$.

Let $G^{\prime}$ be $U(2,2)$ so that $G^{\prime} \times H \subset S p\left(W^{\prime}\right)$, where $W^{\prime}=W \oplus W$ with Hermitian form $\langle\langle\rangle\rangle:,=\langle,\rangle \oplus-\langle$,$\rangle . Then$

$$
(X \oplus X) \bigoplus(Y \oplus Y)
$$

is a complete polarization of $W^{\prime}$. We can choose a basis for $W^{\prime}$ so that, with respect to this decomposition, the Hermitian form $\langle\langle\rangle$,$\rangle is given by the matrix$

$$
\left(\begin{array}{llll} 
& & 1 & \\
& & & 1 \\
1 & & & \\
& 1 & &
\end{array}\right) .
$$

We also have another complete polarization of $W^{\prime}$. Let

$$
U^{d}:=\{(u, u) \mid u \in U\}, U_{d}:=\{(u,-u) \mid u \in U\}
$$

and

$$
W^{d}:=U^{d} \otimes V, W_{d}:=U_{d} \otimes V .
$$

Then $W^{\prime}=W_{d} \oplus W^{d}$.

Let $P^{\prime}$ be the parabolic subgroup of $G^{\prime}$ that stabilizes $U^{d}$ (and hence $W^{d}$ ).

We can embed the product $G \times G$ into $G^{\prime}$ by an embedding $\iota$ such that

$$
\iota\left(g_{1}, g_{2}\right)\left(u_{1}, u_{2}\right)=\left(g_{1} u_{1}, g_{2} u_{2}\right)
$$

for all $g_{i} \in G$ and $u_{i} \in U$. We identify $G \times G$ with its image under $\iota$.

Given a non-trivial additive character $\psi$ of $\mathbb{A} / F$, there is a metaplectic representation, depending on $\psi$, on the metaplectic cover of $S p\left(W^{\prime}\right)(\mathbb{A})$. Here $\mathbb{A}$ denotes the adele ring of $F$. By [GR], Proposition 3.1.1, we have an explicit splitting of the metaplectic cover over $G^{\prime}(\mathbb{A}) \times H(\mathbb{A})$. This splitting is not unique and depends on the choice of $\psi$ and a Hecke character $\gamma$ whose restriction to $F$ is $\omega_{E / F}$, the 
quadratic character of the extension $E / F$ defined by class field theory. Then the metaplectic representation induces, via the splitting, an oscillator representation $\omega=\omega(\psi, \gamma)$ of $G^{\prime}(\mathbb{A}) \times H(\mathbb{A})$. We have

$$
\omega \circ \iota=\omega_{+} \otimes \omega_{-}
$$

where $\omega_{+}$(resp. $\omega_{-}$) is the oscillator representation of $G \times H$ determined by $\psi$ (resp. $\bar{\psi}$ ) and $\gamma$. We can also define similarly the local oscillator representation $\omega_{v}$ (resp. $\omega_{ \pm, v}$ ) of $G_{v}^{\prime} \times H_{v}$ (resp. $G_{v} \times H_{v}$ ) but we often abuse notation by omitting the subscript $v$ from $\omega_{v}$ and $\omega_{ \pm, v}$.

Now, for $\varphi_{1}, \varphi_{2}$ any two Schwartz functions in $S(X)$, the tensor product $\varphi=$ $\varphi_{1} \otimes \bar{\varphi}_{2}$ belongs to $S(X \oplus X)$. We pass from $S(X \oplus X)$ to $S\left(W_{d}\right)$ via the partial Fourier transform $\varphi \longmapsto \varphi^{*}$ where

$$
\varphi^{*}(w)=\int_{X} \psi(2\langle v, y\rangle) \varphi(v+x, v-x) d v .
$$

Here we have identified $w \in W_{d}$ with $(x, y) \in(X \oplus Y)=W$ by mapping $(w,-w)$ to $w$. We then have

$$
\varphi^{*}(0)=\int_{X} \varphi_{1}(v) \bar{\varphi}_{2}(v) d v=\left\langle\varphi_{1}, \varphi_{2}\right\rangle
$$

where $\langle$,$\rangle denotes the inner product in L^{2}(X)$. Also, the two theta series

$$
\sum_{w \in W_{d}(F)} \varphi^{*}(w) \text { and } \sum_{x, y \in X(F)} \varphi(x, y)
$$

agree by the Poisson summation formula.

Now we define the theta lifting $\Theta(\pi)$ for a cuspidal representation $\pi$ of $G$. First of all, we have our usual theta function

$$
\theta^{ \pm}(g, h, \varphi)=\sum_{x \in X(F)} \omega_{ \pm}(g, h) \varphi(x)
$$

where $\varphi \in S(X(\mathbb{A}))$. For a cusp form $f$ of $G$ in $\pi$, we define

$$
\theta_{\varphi}^{ \pm, f}(h):=\int_{G(F) \backslash G(\mathbb{A})} f(g) \theta^{ \pm}(g, h, \varphi) d g .
$$

This function is well defined and slowly increasing on $H(F) \backslash H(\mathbb{A})$. Then $\Theta(\pi)$ is the representation whose space is spanned by the functions $\theta_{\varphi}^{+}, f$ where $\varphi$ runs through $S(X(\mathbb{A}))$ and $f$ runs through the cusp forms in $\pi$.

Let us also recall the standard Langlands $L$-function $L\left(s, \pi, \gamma^{3}\right)$ attached to $\pi$ and twisted by the character $\gamma^{3}$. (For a general exposition of this type of $L$-functions, please refer to [Bor].) It is defined by an Euler product of local $L$-factors over those places $v$ such that $G_{v}=G\left(F_{v}\right)$ is unramified over $F_{v}$ and $\pi_{v}$ is unramified. These local factors are explicitly given as follows:

When $v$ is inert and $\pi_{v} \subseteq \operatorname{Ind}_{B_{v}}^{G_{v}}(\tau)$, the normalized induced representation of $G_{v}$ from the character $\tau$ of $B_{v} \cong G L\left(1, E_{v}\right)$,

$$
L\left(s, \pi_{v}, \gamma_{v}^{3}\right)=L_{E_{v}}\left(s, \tau \gamma_{v}^{3}\right) L_{E_{v}}\left(s, \bar{\tau}^{-1} \gamma_{v}^{3}\right)
$$

(we use a bar to denote the non-trivial Galois automorphism of $E / F$ ).

When $v$ splits in $E$ and $\pi_{v}=\operatorname{Ind}_{B_{v}}^{G_{v}}\left(\chi_{1} \chi_{2}\right)$ where $\chi_{i}$ are characters of $G L\left(1, F_{v}\right)$,

$$
L\left(s, \pi_{v}, \gamma_{v}^{3}\right)=L_{F_{v}}\left(s, \chi_{1} \gamma_{v}^{3}\right) L_{F_{v}}\left(s, \chi_{2} \gamma_{v}^{3}\right) L_{F_{v}}\left(s, \chi_{1}^{-1} \gamma_{v}^{-3}\right) L_{F_{v}}\left(s, \chi_{2}^{-1} \gamma_{v}^{-3}\right)
$$


Here $L_{F_{v}}(s, \chi)=\frac{1}{1-\chi q_{v}^{-s}}$ and is the local Tate $L$-factors associated to the Hecke character $\chi$ of $F$ where $q_{v}$ is the order of the residue field at $F_{v}$.

Via the base change lift from $G$ to $G L(2, E)$ (see [Rog], section 4.2), we can regard $L\left(s, \pi, \gamma^{3}\right)$ as a standard Langlands $L$-function of $G L(2, E)$. More precisely, if $\Pi_{E}$ is the automorphic representation of $G L(2, E)$ corresponding to $\pi$ by base change lift, then

$$
\Pi_{E, v}=\operatorname{Ind}_{B_{2, v}}^{G L\left(2, E_{v}\right)}\left(\left(\begin{array}{cc}
\alpha & \\
& \beta
\end{array}\right) \mapsto \tau(\alpha / \bar{\beta})\right)
$$

when $v$ is inert and

$$
\begin{aligned}
& \Pi_{E, \varpi_{1}}=\operatorname{Ind}_{B_{2, \varpi_{1}}}^{G L\left(2, E_{\varpi_{1}}\right)}\left(\left(\begin{array}{cc}
\alpha & \\
& \beta
\end{array}\right) \mapsto \chi_{1}(\alpha) \chi_{2}(\beta)\right), \\
& \Pi_{E, \varpi_{2}}=\operatorname{Ind}_{B_{2, \varpi_{2}}}^{G L\left(2, E_{\varpi_{2}}\right)}\left(\left(\begin{array}{ll}
\alpha & \\
& \beta
\end{array}\right) \mapsto \chi_{1}^{-1}(\alpha) \chi_{2}^{-1}(\beta)\right)
\end{aligned}
$$

when $v$ splits as $\varpi_{1} \varpi_{2}$ in $E$. Then

$$
L\left(s, \pi, \gamma^{3}\right)=L\left(s, \Pi_{E} \otimes \gamma^{3}\right)
$$

where the right-hand side above is the standard Langlands $L$-function attached to $\Pi_{E} \otimes \gamma^{3}$ (without twist). Therefore, the study of such $L$-function of $U(2)$ reduces to that of $G L(2)$ (over $E$ ) whose analytic properties are more well known. In particular, we know that the possible pole of the standard $L$-function of $G L(2)$ can only occur at $s=1$. Therefore the $L$-function condition of $G$ in Theorem 1.1 can be replaced by that of $G L(2)$.

\section{Regularized Siegel-Weil formula}

In this section, we recall the regularized Siegel-Weil formula for the dual pair $G^{\prime}$ and $H$ which is the key to the proof of our theta lifting problem. Details of this formula can be found in [Tan2]. As is mentioned in the introduction, the regularized Siegel-Weil formula was first formulated (the so-called first term identity) by S. Kudla and S. Rallis in $[\mathrm{KR}]$ for the dual pair $S p(n) \times O(m)$. In the other paper [KRS], a further result (second term identity) was obtained for the pair $S p(2) \times O(4)$. These two papers have provided a prototype for the regularized Siegel-Weil formula in the unitary case.

There are two objects involved in the formula, namely the Siegel-Eisenstein series and the regularized theta integral. Let $I(s)=\operatorname{Ind}_{P^{\prime}}^{G^{\prime}}\left(\gamma^{3}\|\cdot\|^{s}\right)$ be the normalized induced representation of $G^{\prime}(\mathbb{A})$ inducing from the character $\gamma^{3}\|\cdot\|^{s}$ of the Levi factor of $P^{\prime}(\mathbb{A})$. We denote an element in (the space of) $I(s)$ by $\Phi(s)$ or simply $\Phi$.

Now we define the Siegel-Eisenstein series

$$
E(g, s, \Phi)=\sum_{\varepsilon \in P^{\prime}(F) \backslash G^{\prime}(F)} \Phi(\varepsilon g, s)
$$

for $\Phi(s)$ an element in $I(s)$. This series converges absolutely for $\operatorname{Re}(s)>1$ and has a meromorphic continuation to the whole complex plane. It has a simple pole at $s=\frac{1}{2}$. Let

$$
\frac{A_{-1}(g, \Phi)}{s-\frac{1}{2}}+A_{0}(g, \Phi)+\cdots
$$

be the Laurent expansion of $E(g, s, \Phi)$ at $s=\frac{1}{2}$. Then $A_{-1}$ defines an intertwining map from $I\left(\frac{1}{2}\right)$ to $\mathcal{A}\left(G^{\prime}\right)$, the space of automorphic forms on $G^{\prime}$. 
We now turn over to the regularized theta integral. We first define the theta function

$$
\theta(g, h, \varphi)=\sum_{w \in W_{d}(F)} \omega(g, h) \varphi(w)
$$

where $\varphi \in S\left(W_{d}(\mathbb{A})\right)$. We also need to introduce a Hecke operator $z$ of $H_{v}$ for some unramified inert place $v$. This is a locally constant, compactly supported bi $K$-invariant function on $H_{v}$. Given a representation $\sigma$ of $H_{v}$, a Hecke operator $z$ acts on the space of $\sigma$ via

$$
\sigma(z)=\int_{H_{v}} z(h) \sigma(h) d h
$$

If $\sigma$ is unramified, then $z$ acts on the $K$-fixed vectors of $\sigma$ by scalars. More precisely, suppose $\chi$ is some unramified character of the Borel of $H_{v}$ such that

$$
\chi\left(\begin{array}{ccc}
a & 0 & 0 \\
0 & b & 0 \\
0 & 0 & \bar{a}^{-1}
\end{array}\right)=\rho\left(a^{2} b\right)\|a\|^{s}
$$

for some $s \in \mathbb{C}$ and some unitary character $\rho$. If $v^{0}$ is a $K$-fixed vector in $\sigma$, then $\sigma(z) v^{0}=P\left(q^{ \pm s}\right)$ where $P\left(q^{ \pm s}\right)$ is a symmetric polynomial in $q^{ \pm s}$ over $\mathbb{C}$ and $q$ is the residual characteristic of $F_{v}$. (In fact, $z \mapsto P\left(q^{ \pm s}\right)$ defines a one-to-one correspondence between the set of all Hecke operators of $H_{v}$ and $\mathbb{C}\left[q^{s}\right]^{W_{H_{v}}}$ where $W_{H_{v}}$ is the Weyl group of $H_{v}$. This is called the Satake isomorphism.) For our choice of $z$, the corresponding $P\left(q^{ \pm s}\right)$ is

$$
P\left(q^{ \pm s}\right)=q^{s}+q^{-s}-q-q^{-1}
$$

By Corollary 2.3.2 in [Tan2], we have

Lemma 3.1. $\theta(g, h, \omega(z) \varphi)$ is rapidly decreasing on $H(F) \backslash H(\mathbb{A})$ for all $g$ and $\varphi$.

In order to compare our theta integral with the Siegel-Eisenstein series, we need to incorporate an auxiliary Eisenstein series $E(h, s)$ (see [Tan1, section 3.3]) of $H$ into the definition. Then the regularized theta integral is defined by

$$
\mathcal{E}(g, s, \varphi)=\frac{1}{P\left(q^{ \pm s}\right)} \int_{H(F) \backslash H(\mathbb{A})} \theta(g, h, \omega(z) \varphi) E(h, s) \gamma^{-2}(\operatorname{det} h) d h .
$$

$E(h, s)$ has a simple pole at $s=1$ with constant residue. So we may write

$$
E(h, s)=\frac{\kappa}{s-1}+\kappa_{0}(h)+\cdots .
$$

Also $P\left(q^{ \pm s}\right)$ has a zero at $s=1$. So

$$
\frac{1}{P\left(q^{ \pm s}\right)}=\frac{a}{s-1}+b+\cdots
$$

Hence $\mathcal{E}(g, s, \varphi)$ has a double pole at $s=1$. Let us write its Laurent expansion as

$$
\frac{B_{-2}(g, \varphi)}{(s-1)^{2}}+\frac{B_{-1}(g, \varphi)}{(s-1)}+B_{0}(g, \varphi)+\cdots .
$$

In view of (3.2) and (3.3), we have the expressions

$$
B_{-2}(g, \varphi)=a \kappa \int_{H(F) \backslash H(\mathbb{A})} \theta(g, h, \omega(z) \varphi) \gamma^{-2}(\operatorname{det} h) d h
$$


and

$$
B_{-1}(g, \varphi)=\frac{b}{a} B_{-2}(g, \varphi)+a \int_{H(F) \backslash H(\mathbb{A})} \theta(g, h, \omega(z) \varphi) \kappa_{0}(h) \gamma^{-2}(\operatorname{det} h) d h .
$$

Note that the integrals in the above expressions are convergent in view of Lemma 3.1. Again, $B_{-1}$ and $B_{-2}$ define $G^{\prime}(\mathbb{A})$-intertwining maps from $S\left(W_{d}(\mathbb{A})\right)$ to $\mathcal{A}\left(G^{\prime}\right)$.

In order to link the two objects we have just defined, we define a map

such that

$$
\begin{aligned}
S\left(W_{d}(\mathbb{A})\right) & \longrightarrow I\left(\frac{1}{2}\right), \\
\varphi & \mapsto \Phi\left(\frac{1}{2}\right)
\end{aligned}
$$

$$
\Phi\left(\frac{1}{2}\right)(g)=\omega(g) \varphi(0) .
$$

This gives an intertwining map between the two representations. We denote the image of this map by $\Pi(V)$.

If we decompose $V$ as $V_{0} \oplus V_{1,1}$ where $V_{1,1}$ is a hyperbolic plane in $V$, then $V_{0}$ is the one dimensional anisotropic subspace associated to the skew-Hermitian form $\left.(,)^{\prime}\right|_{V_{0}}$. We can define similarly $\Pi\left(V_{0}\right)$ as the space of all $\Phi\left(-\frac{1}{2}\right)$ where

$$
\Phi\left(-\frac{1}{2}\right)(g)=\omega^{\prime}(g) \varphi(0)
$$

and $\omega^{\prime}$ is the oscillator representation of $G^{\prime} \times U(1)$ as $\varphi$ runs over $S\left(U_{d} \otimes V_{0}(\mathbb{A})\right)$.

We may now summarize some of the main results in [Tan2]:

(i) $\operatorname{Im} A_{-1} \cong \bigoplus \Pi\left(V_{00}\right)$ where $V_{00}$ runs over all one dimensional skew Hermitian spaces;

(ii) $\left.\operatorname{Im} A_{-1}\right|_{\Pi(V)} \cong \Pi\left(V_{0}\right)$;

(iii) $\operatorname{Im} B_{-2} \cong \Pi\left(V_{0}\right)$;

(iv) $\Pi\left(V_{0}\right)$ can be embedded in $\mathcal{A}\left(G^{\prime}\right)$ in exactly one way. In particular, we have

(v) (First term identity) There is a non-zero constant $c$ such that, for all $\varphi \in$ $S\left(W_{d}\right)$,

$$
A_{-1}(g, \Phi)=c B_{-2}(g, \varphi)
$$

where $\Phi$ is associated to $\varphi$.

We also have

(vi) (Second term identity) There is a non-zero constant $c$ such that, for all $\varphi \in S\left(W_{d}\right)$,

for some $\Psi$ in $\operatorname{Im} A_{-1}$.

$$
A_{0}(g, \Phi)=c B_{-1}(g, \varphi)+\Psi(g)
$$

\section{The NON-VANishing RESUlT}

Let us consider the integral

$$
\mathcal{Z}\left(s, f_{1}, f_{2}, \Phi\right):=\int_{G(F) \times G(F) \backslash G(\mathbb{A}) \times G(\mathbb{A})} f_{1}\left(g_{1}\right) \bar{f}_{2}\left(g_{2}\right) E\left(\left(g_{1}, g_{2}\right), s, \Phi\right) d g_{1} d g_{2}
$$

where $f_{1}, f_{2} \in \pi, \Phi=\Phi(s) \in I(s)$ and $E\left(g^{\prime}, s, \Phi\right)$ is our Siegel-Eisenstein series.

Using the fundamental identity, which was first introduced in [GPS], we have

$$
\mathcal{Z}\left(s, f_{1}, f_{2}, \Phi\right)=\int_{G(\mathbb{A})} \Phi((g, 1))\left\langle\pi(g) f_{1}, f_{2}\right\rangle d g
$$

where $\left\langle f_{1}, f_{2}\right\rangle=\int_{G(F) \backslash G(\mathbb{A})} \gamma^{3}(\operatorname{det} g) f_{1}(g) \bar{f}_{2}(g) d g$. 
Now suppose $\Phi, f_{1}, f_{2}$ are decomposable as local factors. Then the global zeta integral on the right-hand side of (4.1) admits an Euler product:

$$
\prod_{v} \int_{G_{v}} \Phi_{v}((g, 1))\left\langle\pi_{v}(g) f_{1, v}, f_{2, v}\right\rangle d g .
$$

Our next lemma says that, for almost all $v$, the local zeta integrals in (4.2) are essentially the local factors of the (twisted) Langlands $L$-function associated with $\pi_{v}$ when $\Phi_{v}, f_{1, v}, f_{2, v}$ are chosen properly.

Let $\mathcal{S}$ be a finite set of places in $F$ including all archimedean places such that everything is unramified outside $\mathcal{S}$ (i.e. $G_{v}, \pi_{v}, \gamma_{v}, \psi_{v}$ etc. are unramified).

Then by a tedious but similar computation as in [Li2] (see [Tan1, chapter 6] for details), we obtain:

Lemma 4.3. Let $v \notin \mathcal{S}$. For $\Phi_{v}^{0}$ the normalized $K_{v}^{\prime}$-fixed vector in $I_{v}(s)$ and $f_{0, v}$ a $K_{v}$-fixed vector in $\pi_{v}$ such that $\left\langle f_{0, v}, f_{0, v}\right\rangle=1$,

$$
\int_{G_{v}} \Phi_{v}^{0}((g, 1))\left\langle\pi_{v}(g) f_{0, v}, f_{0, v}\right\rangle d g=\frac{1}{\xi_{v}(s)} L_{v}\left(s+\frac{1}{2}, \pi_{v}, \gamma_{v}^{3}\right)
$$

where $\xi_{v}(s)=L_{F_{v}}(2 s+1) L_{F_{v}}\left(2 s+2, \omega_{E / F}\right)$ and $L_{v}\left(s, \pi_{v}, \gamma_{v}^{3}\right)$ is given by (2.3) and (2.4).

Let us choose two cusp forms $f_{1}, f_{2}$ in $\pi$. For almost all $v$,

$$
f_{1, v}=f_{2, v}=f_{0, v}
$$

where $f_{0, v}$ is as in Lemma 4.3. We may assume (4.4) is satisfied for every $v$ outside $\mathcal{S}$. We also choose $\Phi\left(\frac{1}{2}\right)$ from $\Pi(V)$, i.e. $\Phi\left(\frac{1}{2}\right)\left(g^{\prime}\right)=\omega\left(g^{\prime}\right) \varphi^{*}(0)$ where $\varphi^{*} \in S\left(W_{d}\right)$ is the partial Fourier transform of $\varphi=\varphi_{1} \otimes \bar{\varphi}_{2} \in S(X \oplus X)$. In view of (2.1),

$$
\Phi\left(\frac{1}{2}\right)((g, 1))=\left\langle\omega_{+}(g) \varphi_{1}, \varphi_{2}\right\rangle .
$$

At almost all places, $\Phi_{v}$ is the normalized $K^{\prime}$-fixed vector in $I_{v}(s)$. So we might as well assume that, outside the finite set $\mathcal{S}, \Phi_{v}=\Phi_{v}^{0}$. In particular, for $v \notin \mathcal{S}, \varphi_{1, v}$ and $\varphi_{2, v}$ are the characteristic functions of the lattice $X\left(\mathcal{O}_{E_{v}}\right)$.

We shall examine the analytic property of $\mathcal{Z}\left(s, f_{1}, f_{2}, \Phi\right)$ at the point $s=\frac{1}{2}$. From Lemma 4.3,

$$
\begin{aligned}
\mathcal{Z}\left(\frac{1}{2},\right. & \left.f_{1}, f_{2}, \Phi\right) \\
& =L^{\mathcal{S}}\left(1, \pi, \gamma^{3}\right) \prod_{v \in \mathcal{S}} \int_{G_{v}}\left\langle\omega_{+}(g) \varphi_{1, v}, \varphi_{2, v}\right\rangle\left\langle\pi_{v}(g) f_{1, v}, f_{2, v}\right\rangle d g .
\end{aligned}
$$

where $L^{\mathcal{S}}\left(s, \pi, \gamma^{3}\right)=\prod_{v \notin \mathcal{S}} L_{v}\left(s, \pi_{v}, \gamma_{v}^{3}\right)$ is the partial direct product of the local $L$-factors. By the discussion in section 2, the possible pole of this object can only occur at $s=1$. Whenever $\pi_{v}$ is a discrete series, the local integrals

$$
\int_{G_{v}}\left\langle\omega_{+}(g) \varphi_{1, v}, \varphi_{2, v}\right\rangle\left\langle\pi_{v}(g) f_{1, v}, f_{2, v}\right\rangle d g
$$

converge absolutely. In fact, this is true more generally for $G=U(n)$ and $H=$ $U(m)$ with $n \leq m$ and $\pi$ in the discrete series of $G$ (see [Li1]). Hence

Lemma 4.7. If $L\left(s, \pi, \gamma^{3}\right)$ does not have a pole at $s=1$ and $\pi_{v}$ are discrete for all $v \in \mathcal{S}$, then $\mathcal{Z}\left(s, f_{1}, f_{2}, \Phi\right)$ has no pole at $s=\frac{1}{2}$. 
Now let us recall that $\mathcal{Z}\left(s, f_{1}, f_{2}, \Phi\right)$ is defined by integrating the Eisenstein series $E\left(\left(g_{1}, g_{2}\right), s, \Phi\right)$ against $f_{1}\left(g_{1}\right) \bar{f}_{2}\left(g_{2}\right)$ over $G(F) \times G(F) \backslash G(\mathbb{A}) \times G(\mathbb{A})$. But $E\left(g^{\prime}, s, \Phi\right)$ has a pole at $s=\frac{1}{2}$ with residue $A_{-1}\left(g^{\prime}, \Phi\right)$ (see section 3). Hence the discussion above implies that

$$
\int_{G(F) \times G(F) \backslash G(\mathbb{A}) \times G(\mathbb{A})} f_{1}\left(g_{1}\right) \bar{f}_{2}\left(g_{2}\right) A_{-1}\left(\left(g_{1}, g_{2}\right), \Phi\right) d g_{1} d g_{2}=0 .
$$

We also have $\left.\operatorname{Im} A_{-1}\right|_{\Pi(V)}=\Pi\left(V_{0}\right)$. Thus we have shown that $f_{1} \bar{f}_{2}$ is orthogonal to $\Pi\left(V_{0}\right)$. If we replace $V$ by another three-dimensional skew Hermitian space $V^{*}$, we get a new group $H^{*}$. Since $f_{1}, f_{2}$ are cusp forms of $G$, by repeating the arguments for the dual pair $G^{\prime} \times H^{*}$, we obtain $f_{1} \bar{f}_{2}$ being orthogonal to $\Pi\left(V_{0}^{*}\right)$ where $V_{0}^{*}$ is the complementary one-dimensional subspace of $V^{*}$. In fact, by allowing $V$ to run through all (global) three-dimensional skew-Hermitian spaces, we obtain

Lemma 4.9. Under the same condition as in Lemma 4.7, $f_{1} \bar{f}_{2}$ is orthogonal to $\bigoplus \Pi\left(V_{0}\right)$ where $V_{0}$ ranges over all one-dimensional skew-Hermitian spaces.

Now we turn to the non-vanishing of $\mathcal{Z}\left(\frac{1}{2}, f_{1}, f_{2}, \Phi\right)$. We have to check that the factors in the right-hand side of (4.5) are non-zero. By a well known result of [Jac], the $L$-function $L\left(s, \Pi_{E} \otimes \gamma^{3}\right)$ of $G L(2, E)$ is non-zero at $s=1$. (In fact, this is true for all $G L(n)$ and all $s$ with $\operatorname{Re}(s)=1$.) Therefore, $L\left(s, \pi, \gamma^{3}\right)$ is also non-vanishing at $s=1$.

So it remains to check that the local zeta integrals for $v \in \mathcal{S}$ are also non-zero. Whenever $\pi_{v}$ is discrete and has a theta lifting $\rho_{v}$ in $H_{v}$, it follows from [Li1], section 2 that (4.6) is not identically zero for all $\varphi_{i, v}$ and $f_{i, v}$. Hence we have

Lemma 4.10. Under the same condition as in Lemma 4.7, if $\pi_{v}$ has non-trivial theta-lifting in $H_{v}$ for all $v$, then $\mathcal{Z}\left(s, f_{1}, f_{2}, \Phi\right)$ is holomorphic at $s=\frac{1}{2}$ and nonzero for some choice of $\Phi$ such that $\Phi\left(\frac{1}{2}\right) \in \Pi(V)$ and $f_{1}, f_{2} \in \pi$.

Finally, we shall see how the results we have obtained so far together with the regularized Siegel-Weil formula imply that the theta lift of $\pi$ to $H$ is non-trivial.

The point is to express $\mathcal{Z}\left(\frac{1}{2}, f_{1}, f_{2}, \Phi\right)$ in terms of theta integral associated to $G^{\prime}$ and $H$.

In view of (4.8), we can write $\mathcal{Z}\left(\frac{1}{2}, f_{1}, f_{2}, \Phi\right)$ as

$$
\int_{G(F) \times G(F) \backslash G(\mathbb{A}) \times G(\mathbb{A})} f_{1}\left(g_{1}\right) \bar{f}_{2}\left(g_{2}\right) A_{0}\left(\left(g_{1}, g_{2}\right), \Phi\right) d g_{1} d g_{2}
$$

where $A_{0}(g, \Phi)$ is the second term in the Laurent expansion of $E(g, s, \Phi)$ at $s=\frac{1}{2}$. Now we invoke the second term identity that we have stated in section 3 to pass from the Eisenstein series to the regularized theta integral. We recall that

$$
A_{0}\left(g^{\prime}, \Phi\right)=c B_{-1}\left(g^{\prime}, \varphi^{*}\right)+\Psi\left(g^{\prime}\right)
$$

where $B_{-1}$ is the second term of the regularized theta integral, $c$ is a constant and $\Psi \in \bigoplus \Pi\left(V_{00}\right)$. By Lemma $4.9, \Psi$ is orthogonal to $f_{1} \bar{f}_{2}$. So we get

$$
\mathcal{Z}\left(\frac{1}{2}, f_{1}, f_{2}, \Phi\right)=c \int_{G(F) \times G(F) \backslash G(\mathbb{A}) \times G(\mathbb{A})} f_{1}\left(g_{1}\right) \bar{f}_{2}\left(g_{2}\right) B_{-1}\left(\left(g_{1}, g_{2}\right), \varphi^{*}\right) d g_{1} d g_{2} .
$$


In view of (3.4) and the fact that $B_{-2}\left(g^{\prime}, \varphi^{*}\right)$ belongs to $\Pi\left(V_{0}\right)$ and hence is orthogonal to $f_{1} \bar{f}_{2}, \mathcal{Z}\left(\frac{1}{2}, f_{1}, f_{2}, \Phi\right)$ becomes a double integral

$$
\begin{aligned}
& \int_{G(F) \times G(F) \backslash G(\mathbb{A}) \times G(\mathbb{A})} f_{1}\left(g_{1}\right) \bar{f}_{2}\left(g_{2}\right) \\
& \quad \times \int_{H(F) \backslash H(\mathbb{A})} a \kappa_{0}(h) \gamma^{-2}(\operatorname{det} h) \theta\left(g^{\prime}, h, \omega(z) \varphi^{*}\right) d h d g_{1} d g_{2} .
\end{aligned}
$$

$\theta\left(\left(g_{1}, g_{2}\right), h, \omega(z) \varphi^{*}\right)$ is rapidly decreasing on $H(F) \backslash H(\mathbb{A})$. Furthermore, $f_{1}, f_{2}$ are cusp forms on $G$ and hence also rapidly decreasing on $G(F) \backslash G(\mathbb{A})$. We can then apply Fubini's Theorem to interchange the two integrals. So we get

$$
\begin{aligned}
& \mathcal{Z}\left(\frac{1}{2}, f_{1}, f_{2}, \Phi\right)=\int_{H(F) \backslash H(\mathbb{A})} a \kappa_{0}(h) \gamma^{-2}(\operatorname{det} h) \\
& \quad \times \int_{G(F) \times G(F) \backslash G(\mathbb{A}) \times G(\mathbb{A})} f_{1}\left(g_{1}\right) \bar{f}_{2}\left(g_{2}\right) \theta\left(\left(g_{1}, g_{2}\right), h, \omega(z) \varphi^{*}\right) d g_{1} d g_{2} d h .
\end{aligned}
$$

Now we are only one step from proving the non-vanishing of $\Theta(\pi)$. What we really have to show is that the function $\theta_{\varphi}^{+, f}$ we defined in section 2 is non-zero for some $f$ and $\varphi$. Using our choice of $f_{1}, f_{2}, \varphi_{1}, \varphi_{2}$, we compute that

$$
\begin{aligned}
\theta_{\varphi_{1}}^{+, f_{1}}(h) \theta_{\bar{\varphi}_{2}}^{-, \bar{f}_{2}}(h) & \int_{G(F) \backslash G(\mathbb{A})} \sum_{x \in X(F)} \omega_{+}(g, h) \varphi_{1}(x) f_{1}(g) d g \\
& \times \int_{G(F) \backslash G(\mathbb{A})} \sum_{x \in X(F)} \omega_{-}(g, h) \bar{\varphi}_{2}(x) \bar{f}_{2}(g) d g \\
= & \int_{G(F) \times G(F) \backslash G(\mathbb{A}) \times G(\mathbb{A})} f_{1}\left(g_{1}\right) \bar{f}_{2}\left(g_{2}\right) \sum_{x, y \in X(F)} \omega\left(\left(g_{1}, g_{2}\right), h\right) \varphi(x, y) d g_{1} d g_{2} .
\end{aligned}
$$

If we convolve $\theta_{\varphi_{1}}^{+, f_{1}}(h) \theta_{\bar{\varphi}_{2}}^{-, \bar{f}_{2}}(h)$ with $z^{\prime}$, the Hecke operator in $H_{v}$ corresponding to $z$ under Howe correspondence (see [MVW]), we get precisely the inner integral in (4.11). Therefore, under the conditions of Lemma 4.10, the non-vanishing of $\mathcal{Z}\left(\frac{1}{2}, f_{1}, f_{2}, \Phi\right)$ implies $\theta_{\varphi_{1}}^{+, f_{1}}$ is non-zero and hence $\Theta(\pi)$ is non-trivial.

We hence proved Theorem 1.1.

\section{REFERENCES}

[Bor] A. Borel : Automorphic L-functions. Proc. Sym. in Pure Math. AMS XXXIII, part 2 (1979) 27-61. MR 81m:10056

[GPS] S. Gelbart, I. Piatetski-Shapiro, S. Rallis : Explicit constructions of automorphic L-functions. LNM 1254 Springer-Verlag, New York (1987). MR 89k:11038

[GR] S. Gelbart, J. Rogawski : L-functions and Fourier-Jacobi coefficients for the unitary group U(3). Inven. Math. 105 (1991) 445-472. MR 93b:11059

[GRS1] S. Gelbart, J. Rogawski, D. Soudry : Periods of Cusp Forms and L-packets. C. R. Acad. Sci., Paris 317, Série I (1993), 717-722. MR 94m:11064

[GRS2] S. Gelbart, J. Rogawski, D. Soudry : Endoscopy, theta-liftings, and period integrals for the unitary group in three variables. Annals Math. 145 (1997) 419-476. CMP 97:14

[Jac] H. JACQUET : A non-vanishing theorem for zeta-functions of $G L_{n}$. Invent. Math. 38 (1976) 1-16. MR 55:5583

[KR] S. Kudla, S. Rallis : A regularized Siegel-Weil formula: the first term identity. Annals Math. 140 (1994) 1-80. MR 95f:11036 
[KRS] S. Kudla, S. Rallis, D. Soudry : On the degree 5 L-function for $S p$ (2). Inventiones Math. 107 (1992) 483-541. MR 93b:11061

[Li1] J. S. LI : Theta lifting for unitary representations with nonzero cohomology. Duke Math. J. 61 (1990) 913-937. MR 92f:22024

[Li2] J. S. LI : Non-vanishing theorems for the cohomology of certain arithmatic quotients. J. reine angew. Math. 428 (1992) 177-217. MR 93e:11067

[MVW] C. Moeglin, M.-F. Vigneras, J.-L. Waldspurger : Correspondence de Howe sur un corps p-adique. LNM 1291 Springer-Verlag, New York (1987). MR 91f:11040

[Rog] J. RogaWsKI : Automorphic representations of Unitary groups in three variables. Annals of Mathematics Studies (1990). MR 91k:22037

[Tan1] V. TAN : A regularized Siegel-Weil formula on unitary groups. Thesis, UCLA (1996).

[Tan2] V. TAN : A regularized Siegel-Weil formula on $U(2,2)$ and $U(3)$. Duke Math. J. 94 (1998) 341-378. CMP 98:16

Department of Mathematics, National University of Singapore, 10 Kent Ridge CresCENT, Singapore 119260

E-mail address: mattanv@nus.edu.sg 